Ebisu Ebisu

Études japonaises Études japonaises

49 | printemps-été 2013

De chose en fait : la question du milieu

\title{
Témoignage. Les souvenirs de M. Sōkejima
}

Enquête sur le séminaire tenu autour de Yamanouchi Tokuryū à ShinHirayu

Inside Look. The Memories of Mr Sōkejima

\section{Kumi Eguchi}

\section{(2) OpenEdition}

12 Journals

Édition électronique

URL : http://journals.openedition.org/ebisu/711

DOI : 10.4000/ebisu.711

ISSN : 2189-1893

Éditeur :

Institut français de recherche sur le Japon (UMIFRE 19 MAEE-CNRS), Maison franco-japonaise

\section{Édition imprimée}

Date de publication : 1 avril 2013

Pagination : $37-40$

ISSN : 1340-3656

\section{Référence électronique}

Kumi Eguchi, «Témoignage. Les souvenirs de M. Sōkejima », Ebisu [En ligne], 49 | printemps-été 2013, mis en ligne le 21 mars 2014, consulté le 02 mai 2019. URL : http://journals.openedition.org/ ebisu/711 ; DOI : 10.4000/ebisu.711 


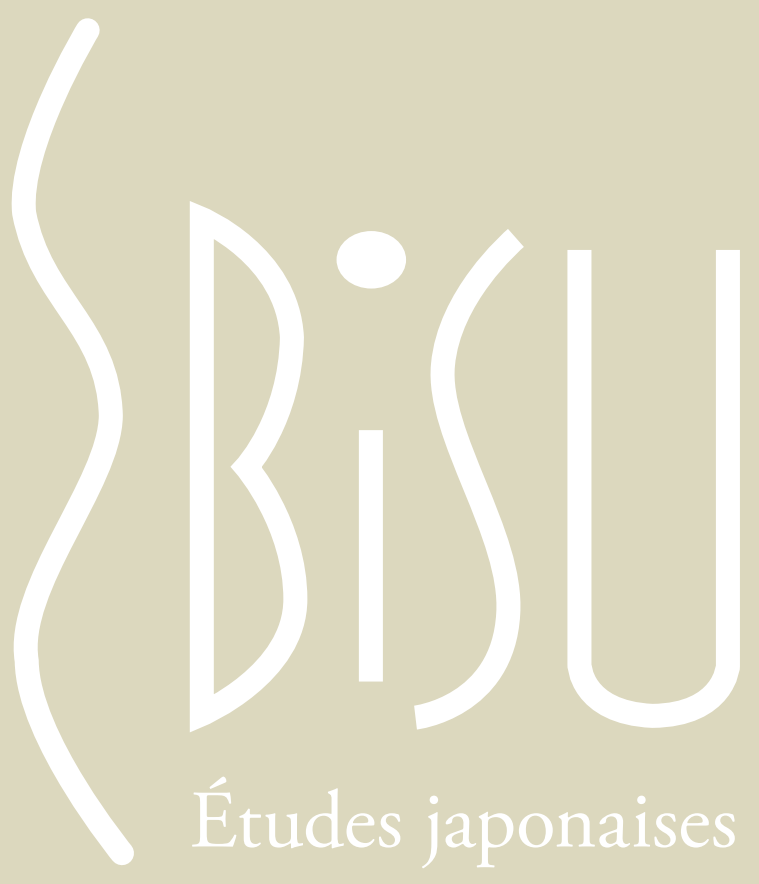

Dossier

De chose en fait : la question du milieu

Articles issus du colloque de Shin-Hirayu

Coordonné par Augustin BERQue

Varia | Nicolas Baumert - Peut-il exister des terroirs du saké ?

Conférences de la MFJ | François Macé - Le Kojiki, une Énéide longtemps oubliée ? | Patrick Beillevaire - Présences françaises à Okinawa : de Forcade (1844-1846) à Haguenauer (1930)

Livres à lire

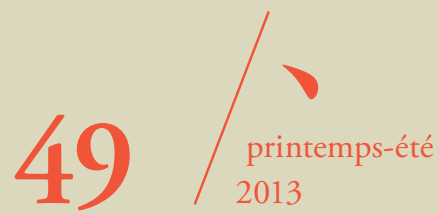




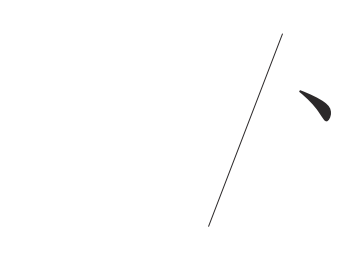

\section{Dossier}

\section{De chose en fait : la question du milieu}

Mono kara koto e to füdo no mondai

物から事へと風土の問題

Articles issus $d u$

colloque de Shin-Hirayu

Dossier coordonné

par Augustin Berque 


\section{Témoignage}

\section{Les souvenirs de M. Sōkejima}

\section{Enquête sur le séminaire tenu autour de}

Yamanouchi Tokuryū à Shin-Hirayu

\section{EGUCHI Kumi 江口久美*}

\section{Prologue}

Quand Augustin Berque a reçu le livre de Yamanouchi Tokuryū Logos et Lemme (Rogosu to Renma ロゴスとレンマ) de la part de Nakamura Yoshio, qui l'avait acheté chez un bouquiniste à Kanda, il y a trouvé un prospectus disant ce qui suit :

«Invitation pour le séminaire autour de Yamanouchi Tokuryū, philosophe et professeur honoraire de l'université de Kyoto. Thème : «Les problèmes de la philosophie chez Nishida. Des choses aux faits, le problème de la causalité ». Date : du 29 juillet au 2 août. Lieu : Shin-Hirayu onsen, ville de Kamitakara, canton de Yoshiki, département de Gifu (ville d'eaux d'Okuhida dans les Alpes du nord). Frais : 19000 yen »

哲学者山内得立氏(京大名誉教授)を囲んでの合宿ゼミナ一ルの案内(中略)。講座の題： 『西田哲学の諸問題』物から事へと故との問題。日時: 7 月 29 日 8 月 2 日 ( 4 泊 5 日)。 場所：岐皁県吉城郡上宝村新平湯温泉（北アルプス奥飛騨温泉郷）。費用：19,000 円

Plus bas dans le prospectus figurait le nom de Sōkejima Shigeru 篮島茂 qui était le président du Groupe de recherches philosophiques de l'université Waseda (Waseda daigaku tetsugaku kenkyūkai 早稲田大学哲学研究会) et l'organisateur de la rencontre.

* Post-doctorante, Graduate School of Human and Environmental Studies, université de Kyoto. Chercheure invitée RCAST (Urban Conservation Systems), université de Tokyo. 


\section{Prendre contact avec Sōkejima Shigeru}

Ce ne fut pas facile de contacter Sōkejima. Bien sûr, le numéro de téléphone qui était inscrit sur le prospectus n'était plus d'actualité. J'ai essayé de le trouver dans les pages jaunes, sans succès. Alors que j'étais sur le point de renoncer, ma mère m’a donné une idée tirée d'un roman policier. En effet, dans Vase de sable (Suna no utsuwa 砂の器) de Matsumoto Seichō 松本清張, l'inspecteur commence ses recherches dans la région où le patronyme est le plus répandu. De la même façon, j'ai découvert que de nombreux «Sōkejima " vivaient dans le département de Toyama. J'ai repris mes recherches à partir des pages jaunes de ce département, et ai finalement réussi à contacter son père qui m’a donné ses coordonnées. J'ai réalisé un entretien avec Sōkejima depuis Paris le 31 janvier 2012.

\section{La carrière de Sōkejima}

Sōkejima Shigeru est né à Toyama. Il était à l'époque du séminaire de ShinHirayu étudiant de la faculté d'économie de l'École des sciences politiques et d'économie de l'université Waseda. Ensuite, il est entré à l'université de médecine de Toyama et s'est spécialisé en « hygiène publique ». Il a travaillé comme maitre de conférences à Toyama, puis à la faculté de médecine de l'université de Kyoto. Plus tard, il a travaillé à l'institut de recherche du ministère de la Santé, du Travail et des Affaires sociales. Depuis 2010, il est professeur à l'école doctorale de médecine de l'université de Mie.

\section{La relation entre Sōkejima et Yamanouchi}

Yamanouchi appréciait beaucoup la montagne. Il a notamment passé du temps dans les Alpes quand il étudiait en Allemagne. Un ami du père de Sōkejima était élève de Yamanouchi. Le père de Sōkejima a organisé un séjour de vacances d'été pour Yamanouchi et son épouse dans un hôtel à Tengudaira 天狗平 situé sur les flancs du mont Tateyama 立山à Toyama. C'est grâce à ces liens, en 縁, que Sōkejima a fait connaissance avec Yamanouchi. Tengudaira est un plateau situé à 2000 mètres d'altitude. Yamanouchi s'y est longuement promené alors qu'il profitait du séjour pour écrire un article, probablement Zuimen no tetsugaku 随眠の哲学 (Philosophie de la latence [anuśaya]), Iwanami shoten 岩波書店, 1993). Il a beaucoup aimé le lieu et disait : «La montagne que je vois depuis l'hôtel est plus belle que les 
Alpes. » Le père de Sōkejima était spécialiste de droit, mais il s’intéressait beaucoup à la philosophie et s'est lié d'amitié avec Yamanouchi. À l'époque, Sōkejima était dans le domaine de l'économie mais s'intéressait également à la philosophie. Il était le président du Groupe de recherches philosophiques de l'université Waseda, qui comptait plusieurs dizaines de membres. Quand il a rencontré Yamanouchi pour la première fois, ce dernier lui a beaucoup parlé de Hegel. Il lui a conseillé d'étudier avec Kashiyama Kinshirō 樫山 欽四郎 (1907-1977) qui était professeur à l’université Waseda. Kashiyama fut le premier hégélien, reconnu pour ses études minutieuses du philosophe allemand. Cependant, à l'époque, Kashimaya se portait mal et il fut impossible pour Sōkejima d'étudier avec lui.

\section{Le séminaire de Shin-Hirayu}

Sōkejima a organisé un séminaire en invitant Yamanouchi vers 1980, en tant que président du Groupe de recherches philosophiques. Le but du séminaire était de permettre à une dizaine des membres du groupe d'écouter le cours de Yamanouchi tout en séjournant dans une auberge à Shin-Hirayu onsen au pied du mont Hotaka 穂高岳 à Gifu. Beaucoup des participants étaient des étudiants du département de philosophie, d'autres venaient des départements de droit ou d'économie, tel Sōkejima. Ils entretenaient de proches relations, bien que d'âges sensiblement différents.

Le thème du séminaire, "Les problèmes de la philosophie chez Nishida. Des choses aux faits, le problème de la causalité ", est issu de l'ouvrage Logos et Lemme. À Shin-Hirayu, Yamanouchi a donné un cours dans un langage accessible et a peut-être dévoilé ses véritables pensées, car c'était un cours privé pour les étudiants présents. D’après les souvenirs de Sōkejima, Yamanouchi avait parlé de Parménide, car il était spécialiste des philosophes grecs présocratiques. Yamanouchi avait aussi traité de Heidegger afin d'aborder le "problème du logos ».

Le séminaire n’a pas été le lieu de discussions, mais a consisté en un cours par Yamanouchi seul, que les étudiants écoutaient, posant parfois quelques questions. Les journées de cours furent ponctuées de promenades en groupe sur le piémont du Hodaka.

Sōkejima n'a pas pu retrouver la cassette où il avait enregistré ce séminaire, faute de temps pour se rendre à Toyama, mais espère pouvoir un jour mettre la main dessus. 
Pour finir, il se souvient de ces mots de Yamanouchi : «Le plus important dans la philosophie, c'est de continuer, et de persévérer dans la même direction longtemps. » 